\title{
On the Continuity Properties of the Attainable Sets of Nonlinear Control Systems with Integral Constraint on Controls
}

\author{
Khalik G. Guseinov and Ali S. Nazlipinar \\ Department of Mathematics, Anadolu University, Eskisehir 26470, Turkey \\ Correspondence should be addressed to Khalik G. Guseinov, kguseynov@anadolu.edu.tr
}

Received 11 June 2007; Revised 30 August 2007; Accepted 6 November 2007

Recommended by Agacik Zafer

The attainable sets of the nonlinear control systems with integral constraint on the control functions are considered. It is assumed that the behavior of control system is described by a differential equation which is nonlinear with respect to phase-state vector and control vector. The admissible control functions are chosen from the closed ball centered at the origin with radius $\mu_{0}$ in $L_{p}\left(\left[t_{0}, \theta\right] ; \mathbb{R}^{m}\right)(p \in(1,+\infty))$. Precompactness of the solutions set is specified, and dependence of the attainable sets on the initial conditions and on the parameters of the control system is studied.

Copyright ( 2008 K. G. Guseinov and A. S. Nazlipinar. This is an open access article distributed under the Creative Commons Attribution License, which permits unrestricted use, distribution, and reproduction in any medium, provided the original work is properly cited.

\section{Introduction}

Control problems with integral constraints on control arise in various problems of mathematical modeling. For example, the motion of flying apparatus with variable mass is described in the form of controllable system, where the control function has integral constraints (see, e.g., [1-3]). One of the important constructions of the control systems theory is the attainable set notion. Attainable set is the set of all points to which the system can be steered at the instant of given time. Attainable sets of control systems are very useful tools in the study of various problems of optimization, dynamical systems and differential game theory.

In [4-10], topological properties and numerical construction methods of the attainable sets of linear control systems with integral constraint on control functions are investigated. The attainable sets of affine control systems, that is, the attainable sets of control systems which are nonlinear with respect to the phase-state vector, but are linear with respect to the control vector have been considered in [11-14]. The properties of the attainable sets of the nonlinear control systems have been studied in [15-18].

Approximation method for the construction of attainable sets of affine control systems with integral constraints on the control is given in [11, 13]. In [14], using the topological 
properties of attainable sets of affine control systems, the continuity properties of minimum time and minimum energy functions are discussed.

The dependence of the attainable set on $p$ is studied in $[8,12,15]$. In [15], it is proved that attainable set of affine control system depends on $p$ continuously. In [15], the same property is shown for nonlinear control systems.

In [17], if the control resource is sufficiently small, then under some suitable assumptions on the right-hand side of the system, it is proved that the attainable set of the nonlinear control system with integral constraints on control is convex.

The value function of nonlinear optimal control problem with generalized integral constraints on control and phase-state vectors is investigated in $[16,18]$.

In this article, we consider the attainable sets of the control systems the behavior of which is described by nonlinear differential equations. It is assumed that the admissible control functions are chosen from the closed ball centered at the origin with radius $\mu_{0}$ in $L_{p}\left(\left[t_{0}, \theta\right] ; \mathbb{R}^{m}\right)(p \in(1,+\infty))$.

In Section 2, it is illustrated that, in general, the attainable set is not closed (Example 2.5) and it is shown that the set of solutions generated by all possible admissible control functions is precompact in the space of continuous functions (Corollary 2.4). In Section 3, the diameter of the attainable set is evaluated (Proposition 3.1) and it is proved that the attainable set is Hölder continuous with respect to time variable (Proposition 3.3). In Section 4, it is shown that the attainable set of the control system is continuous with respect to initial condition (Proposition 4.1). In Section 5, it is proved that the attainable set is Lipschitz continuous with respect to a parameter of the system which define the resource of the control effort (Proposition 5.1).

Consider the control system the behavior of which is described by the differential equation

$$
\dot{x}(t)=f(t, x(t), u(t)), \quad x\left(t_{0}\right) \in X_{0},
$$

where $x \in \mathbb{R}^{n}$ is the phase-state vector of the system, $u \in \mathbb{R}^{m}$ is the control vector, $t \in\left[t_{0}, \theta\right]$ is the time, and $X_{0} \subset \mathbb{R}^{n}$ is a compact set.

For $p \in(1, \infty)$ and $\mu_{0}>0$, we set

$$
U_{p}^{\mu_{0}}=\left\{u(\cdot) \in L_{p}\left(\left[t_{0}, \theta\right], \mathbb{R}^{m}\right):\|u(\cdot)\|_{p} \leq \mu_{0}\right\},
$$

where $\|u(\cdot)\|_{p}=\left(\int_{t_{0}}^{\theta}\|u(t)\|^{p} d t\right)^{1 / p}$ and $\|\cdot\|$ denotes the Euclidian norm.

A function $u(\cdot) \in U_{p}^{\mu_{0}}$ is said to be an admissible control function. It is obvious that the set of all admissible control functions $U_{p}^{\mu_{0}}$ is the closed ball centered at the origin with the radius $\mu_{0}$ in $L_{p}\left(\left[t_{0}, \theta\right] ; \mathbb{R}^{m}\right)$. tions.

It is assumed that the right-hand side of the system (1.1) satisfies the following condi-

(a) The function $f(\cdot):\left[t_{0}, \theta\right] \times \mathbb{R}^{n} \times \mathbb{R}^{m} \rightarrow \mathbb{R}^{n}$ is continuous.

(b) For any bounded set $D \subset\left[t_{0}, \theta\right] \times \mathbb{R}^{n}$, there exist constants $L_{1}=L_{1}(D)>0, L_{2}=L_{2}(D)>0$, and $L_{3}=L_{3}(D)>0$ such that

$$
\left\|f\left(t, x_{1}, u_{1}\right)-f\left(t, x_{2}, u_{2}\right)\right\| \leq\left(L_{1}+L_{2}\left\|u_{2}\right\|\right)\left\|x_{1}-x_{2}\right\|+L_{3}\left\|u_{1}-u_{2}\right\|
$$

for any $\left(t, x_{1}\right) \in D,\left(t, x_{2}\right) \in D, u_{1} \in \mathbb{R}^{m}$, and $u_{2} \in \mathbb{R}^{m}$. 
(c) There exists a constant $c>0$ such that

$$
\|f(t, x, u)\| \leq c(1+\|x\|)(1+\|u\|)
$$

for every $(t, x, u) \in\left[t_{0}, \theta\right] \times \mathbb{R}^{n} \times \mathbb{R}^{m}$.

If the right-hand side of the system (1.1) is affine, that is, if $f(t, x, u)=\varphi(t, x)+B(t, x) u$ and the functions $\varphi(\cdot):\left[t_{0}, \theta\right] \times \mathbb{R}^{n} \rightarrow \mathbb{R}^{n}, B(\cdot):\left[t_{0}, \theta\right] \times \mathbb{R}^{n} \rightarrow \mathbb{R}^{m}$ satisfy the assumptions given in [11-14], then, under these assumptions, the conditions (a), (b), and (c) are also fulfilled.

Let $u_{*}(\cdot) \in U_{p}^{\mu_{0}}$. The absolutely continuous function $x_{*}(\cdot):\left[t_{0}, \theta\right] \rightarrow \mathbb{R}^{n}$, which satisfies the equation $\dot{x}_{*}(t)=f\left(t, x_{*}(t), u_{*}(t)\right)$ a.e. in $\left[t_{0}, \theta\right]$, and the initial condition $x_{*}\left(t_{0}\right)=x_{0} \in X_{0}$ is said to be a solution of the system (1.1) with initial condition $x_{*}\left(t_{0}\right)=x_{0}$, generated by the admissible control function $u_{*}(\cdot)$. By the symbol $x\left(\cdot ; t_{0}, x_{0}, u(\cdot)\right)$, we denote the solution of the system (1.1) with initial condition $x\left(t_{0}\right)=x_{0}$, which is generated by the admissible control function $u(\cdot)$. Note that the conditions (a)-(c) guarantee the existence, uniqueness, and extendability of the solutions up to the instant of time $\theta$ for every given $u_{*}(\cdot) \in U_{p}^{\mu_{0}}$ and $x_{0} \in X_{0}$.

Let us define the sets

$$
\begin{aligned}
X_{p}\left(t_{0}, X_{0}, \mu_{0}\right) & =\left\{x\left(\cdot ; t_{0}, x_{0}, u(\cdot)\right):\left[t_{0}, \theta\right] \longrightarrow \mathbb{R}^{n} \mid x_{0} \in X_{0}, u(\cdot) \in U_{p}^{\mu_{0}}\right\}, \\
X_{p}\left(t ; t_{0}, X_{0}, \mu_{0}\right) & =\left\{x(t) \in \mathbb{R}^{n}: x(\cdot) \in X_{p}\left(t_{0}, X_{0}, \mu_{0}\right)\right\},
\end{aligned}
$$

where $t \in\left[t_{0}, \theta\right]$.

The set $X_{p}\left(t ; t_{0}, X_{0}, \mu_{0}\right)$ is called the attainable set of the system (1.1) at the instant of time $t$. It is obvious that the set $X_{p}\left(t ; t_{0}, X_{0}, \mu_{0}\right)$ consists of all $x \in \mathbb{R}^{n}$ to which the system (1.1) can be steered at the instant of time $t \in\left[t_{0}, \theta\right]$.

The Hausdorff distance between the sets $A \subset \mathbb{R}^{n}$ and $E \subset \mathbb{R}^{n}$ is denoted by $h(A, E)$ and is defined as

$$
h(A, E)=\max \left\{\sup _{x \in A} d(x, E), \sup _{y \in E} d(y, A)\right\},
$$

where $d(x, E)=\inf \{\|x-y\|: y \in E\}$.

By $C\left(\left[t_{0}, \theta\right] ; \mathbb{R}^{n}\right)$, we denote the space of continuous functions $x(\cdot):\left[t_{0}, \theta\right] \rightarrow \mathbb{R}^{n}$ with norm

$$
\|x(\cdot)\|_{C}=\max _{t \in\left[t_{0}, \theta\right]}\|x(t)\| .
$$

Also, $h_{C}(U, V)$ denotes the Hausdorff distance between the sets $U \subset C\left(\left[t_{0}, \theta\right] ; \mathbb{R}^{n}\right)$ and $V \subset C\left(\left[t_{0}, \theta\right] ; \mathbb{R}^{n}\right)$.

\section{Precompactness of the set of solutions}

The following proposition asserts that the set of solutions and the attainable sets of the control system (1.1) with constraint (1.2) are bounded.

Proposition 2.1. Let $p \in(1, \infty), \mu_{*} \in\left(0, \mu_{0}+1\right), h\left(X_{0}, X_{*}\right) \leq 1$. Then for any $x_{*}(\cdot) \in X_{p}\left(t_{0}, X_{*}, \mu_{*}\right)$, the inequality

$$
\left\|x_{*}(\cdot)\right\|_{C} \leq r_{*}
$$


holds, where

$$
\begin{aligned}
r_{*} & =d_{1} \exp (k), \\
d_{1} & =1+d_{*}+k, \\
d_{*} & =\max \left\{\|x\|: x \in X_{0}\right\}, \\
k & =c\left[\left(\theta-t_{0}\right)+l_{*}\left(\mu_{0}+1\right)\right], \\
l_{*} & =\max \left\{\left(\theta-t_{0}\right), 1\right\},
\end{aligned}
$$

and $c>0$ is the constant given in condition (c).

The proof of the proposition follows from condition (c) and Gronwall's inequality. For given $\gamma>0$, we set

$$
\begin{aligned}
D(\gamma) & =\left\{(t, x) \in\left[t_{0}, \theta\right] \times \mathbb{R}^{n}:\|x\| \leq \gamma\right\}, \\
B_{n}(\gamma) & =\left\{x \in \mathbb{R}^{n}:\|x\| \leq \gamma\right\} .
\end{aligned}
$$

We get from Proposition 2.1 that $(t, x(t)) \in D\left(r_{*}\right)$ for every $p \in(1, \infty), x(\cdot) \in X_{p}\left(t_{0}\right.$, $\left.X_{*}, \mu_{*}\right), t \in\left[t_{0}, \theta\right], \mu_{*} \in\left(0, \mu_{0}+1\right)$, and compact $X_{*} \subset \mathbb{R}^{n}$ such that $h\left(X_{0}, X_{*}\right) \leq 1$. So, we have the validity of the following corollary.

Corollary 2.2. The set $X_{p}\left(t_{0}, X_{0}, \mu_{0}\right)$ is uniformly bounded, and consequently $X_{p}\left(t ; t_{0}, X_{0}\right.$, $\left.\mu_{0}\right) \subset B_{n}\left(r_{*}\right)$ for every $t \in\left[t_{0}, \theta\right]$, where $r_{*}$ is defined by (2.2).

Here and henceforth, we will have in mind the cylinder $D\left(r_{*}\right)$ as the set $D$ in condition (b). We set also

$$
k_{*}=c\left(1+r_{*}\right)\left(l_{*}+\mu_{0}\right),
$$

where $r_{*}$ is defined by (2.2), $l_{*}$ is defined by (2.4).

Proposition 2.3. The set $X_{p}\left(t_{0}, X_{0}, \mu_{0}\right)$ is equicontinuous.

Proof. Let $\varepsilon>0$ be an arbitrarily given number. Now, let us choose an arbitrary $x(\cdot) \in$ $X_{p}\left(t_{0}, X_{0}, \mu_{0}\right)$ and $t_{1}, t_{2} \in\left[t_{0}, \theta\right]$. Without loss of generality, we assume that $t_{1} \leq t_{2}$. Then from condition (c), we have

$$
\left\|x\left(t_{1}\right)-x\left(t_{2}\right)\right\| \leq \int_{t_{1}}^{t_{2}} c(1+\|x(\tau)\|)(1+\|u(\tau)\|) d \tau .
$$

According to Proposition 2.1, $\|x(\cdot)\|_{C} \leq r_{*}$, where $r_{*}$ is defined by (2.2). Then we get from (2.4), (2.6), (2.7), and Hölder's inequality that

$$
\begin{aligned}
\left\|x\left(t_{1}\right)-x\left(t_{2}\right)\right\| & \leq c\left(1+r_{*}\right)\left(\left|t_{2}-t_{1}\right|+\mu_{0}\left|t_{2}-t_{1}\right|^{(p-1) / p}\right) \\
& \leq\left|t_{2}-t_{1}\right|^{(p-1) / p} c\left(1+r_{*}\right)\left(\left(\theta-t_{0}\right)^{1 / p}+\mu_{0}\right) \\
& \leq\left|t_{2}-t_{1}\right|^{(p-1) / p} c\left(1+r_{*}\right)\left(l_{*}+\mu_{0}\right)=k_{*}\left|t_{2}-t_{1}\right|^{(p-1) / p} .
\end{aligned}
$$


Thus for given $\varepsilon>0$, setting $\delta(\varepsilon)=\left(\varepsilon / k_{*}\right)^{p /(p-1)}$, we obtain $\left\|x\left(t_{1}\right)-x\left(t_{2}\right)\right\|<\varepsilon$ for $\left|t_{1}-t_{2}\right|<\delta(\varepsilon)$. Since $x(\cdot) \in X_{p}\left(t_{0}, X_{0}, \mu_{0}\right)$ is arbitrarily chosen, the equicontinuity of the set $X_{p}\left(t_{0}, X_{0}, \mu_{0}\right)$ follows.

From Corollary 2.2 and Proposition 2.3, we get the validity of the following corollary.

Corollary 2.4. The set $X_{p}\left(t_{0}, X_{0}, \mu_{0}\right)$ is a precompact subset of the space $C\left(\left[t_{0}, \theta\right], \mathbb{R}^{n}\right)$.

Note that if the right-hand side of the system (1.1) is affine with respect to the control vector $u$, then the weak compactness of the set of admissible control functions $U_{p}^{\mu_{0}}$ guaranties the closeness of the attainable sets; but the attainable sets of the control system (1.1) with constraint (1.2), in general, are not closed. In [19, 20], the example is given which illustrates that the attainable set of nonlinear control system with geometric constraint on control is not closed. We use that example to show that the attainable set of nonlinear control system with integral constraint on control is not also closed.

Example 2.5. Let us consider the control system

$$
\begin{gathered}
\dot{x}=-y^{2}+u^{2}, \quad x(0)=0, \\
\dot{y}=u, \quad y(0)=0,
\end{gathered}
$$

where $(x, y) \in \mathbb{R}^{2}$ is the phase-state vector of the system, $u \in \mathbb{R}$ is the control vector, $t \in[0,1]$. It is assumed that $\mu_{0}=1$ and the control function $u(\cdot) \in L_{2}([0,1] ; \mathbb{R})$ of the system (2.9) satisfies the integral constraint

$$
\int_{0}^{1} u^{2}(t) d t \leq 1
$$

that is, $u(\cdot) \in U_{2}^{1}$. Let us denote

$$
\begin{aligned}
X_{2}(0,(0,0), 1) & =\left\{(x(\cdot ; 0,(0,0), u(\cdot)), y(\cdot ; 0,(0,0), u(\cdot))): u(\cdot) \in U_{2}^{1}\right\}, \\
X_{2}(t ; 0,(0,0), 1) & =\left\{(x(t), y(t)) \in \mathbb{R}^{2}:(x(\cdot), y(\cdot)) \in X_{2}(0,(0,0), 1)\right\} .
\end{aligned}
$$

Thus $X_{2}(0,(0,0), 1)$ is the set of solutions, $X_{2}(t ; 0,(0,0), 1)$ is the attainable set of the control system (2.9) at the instant of time $t \in[0,1]$, generated by control functions $u(\cdot) \in U_{2}^{1}$.

Now, let us prove that the solution set $X_{2}(0,(0,0), 1)$ is bounded. Let $(x(\cdot), y(\cdot)) \in$ $X_{2}(0,(0,0), 1)$ be an arbitrarily chosen solution of the system (2.9) with integral constraint (2.10). Then there exists $u(\cdot) \in U_{2}^{1}$ such that

$$
\begin{gathered}
x(t)=\int_{0}^{t}-y^{2}(\tau) d \tau+\int_{0}^{t} u^{2}(\tau) d \tau, \\
y(t)=\int_{0}^{t} u(\tau) d \tau
\end{gathered}
$$

for any $t \in[0,1]$. From (2.10), (2.13), and Hölder's inequality, the inequality

$$
|y(t)| \leq \int_{0}^{t}|u(\tau)| d \tau \leq\left(\int_{0}^{t} 1^{2} d \tau\right)^{1 / 2}\left(\int_{0}^{t}|u(\tau)|^{2} d \tau\right)^{1 / 2} \leq \sqrt{t} \leq 1
$$


holds for all $t \in[0,1]$. Then we get from (2.10), (2.12), and (2.14) that

$$
|x(t)| \leq \int_{0}^{t}|y(\tau)|^{2} d \tau+\int_{0}^{t}|u(\tau)|^{2} d \tau \leq \int_{0}^{t} \tau d \tau+1=1+\frac{t^{2}}{2} \leq \frac{3}{2}
$$

for all $t \in[0,1]$.

However, (2.14) and (2.15) imply that

$$
\|(x(\cdot), y(\cdot))\|_{C}=\max _{t \in[0,1]}\|(x(t), y(t))\|=\max _{t \in[0,1]} \sqrt{x^{2}(t)+y^{2}(t)} \leq \sqrt{1+\frac{9}{4}}<2 .
$$

Since $(x(\cdot), y(\cdot)) \in X_{2}(0,(0,0), 1)$ is arbitrarily chosen, we get that the set $X_{2}(0,(0,0), 1)$ is bounded.

Now we prove that $(1,0) \notin X_{2}(1 ; 0,(0,0), 1)$. Let us assume the contrary, that is, let $(1,0) \in$ $X_{2}(1 ; 0,(0,0), 1)$. Then there exists $\left(x_{*}(\cdot), y_{*}(\cdot)\right) \in X_{2}(0,(0,0), 1)$ such that

$$
x_{*}(1)=1, \quad y_{*}(1)=0 .
$$

Since $\left(x_{*}(\cdot), y_{*}(\cdot)\right) \in X_{2}(0,(0,0), 1)$, then there exists $u_{*}(\cdot) \in U_{2}^{1}$ such that

$$
\begin{gathered}
x_{*}(t)=\int_{0}^{t}-y_{*}^{2}(\tau) d \tau+\int_{0}^{t} u_{*}^{2}(\tau) d \tau, \\
y_{*}(t)=\int_{0}^{t} u_{*}(\tau) d \tau
\end{gathered}
$$

for all $t \in[0,1]$.

From (2.17), (2.18), and (2.19), it follows that

$$
x_{*}(1)=\int_{0}^{1} u_{*}^{2}(\tau) d \tau-\int_{0}^{1}\left(\int_{0}^{\tau} u_{*}(s) d s\right)^{2} d \tau=1 .
$$

Since $u_{*}(\cdot) \in U_{2}^{1}$, then it follows from (2.20) that $u_{*}(t)=0$ for almost all $t \in[0,1]$. Then we have from (2.18) and (2.19) that $x_{*}(t)=0, y_{*}(t)=0$ for every $t \in[0,1]$, which contradicts (2.17). Thus

$$
(1,0) \notin X_{2}(1 ; 0,(0,0), 1) .
$$

Let us show that $(1,0) \in \operatorname{cl}\left(X_{2}(1 ; 0,(0,0)), 1\right)$.

Let $\Gamma^{k}=\{0,1 / 2 k, 2 / 2 k, \ldots,(2 k-1) / 2 k, 1\}$ be a uniform partition of the closed interval $[0,1]$, where $k=1,2, \ldots$ Now we define a sequence of functions $\left\{u_{k}(\cdot)\right\}_{k=1}^{\infty}$, setting

$$
u_{k}(t)= \begin{cases}1, & t \in\left[\frac{2 i}{2 k}, \frac{2 i+1}{2 k}\right), \\ -1, & t \in\left[\frac{2 i+1}{2 k}, \frac{2 i+2}{2 k}\right),\end{cases}
$$

where $i=0,1, \ldots, k-1$. 
It is obvious that $u_{k}(\cdot) \in U_{2}^{1}$ for all $k=1,2, \ldots$ Let $\left(x_{k}(\cdot), y_{k}(\cdot)\right) \in X_{2}(0,(0,0), 1)$ be the solution of the system (2.9) generated by the admissible control function $u_{k}(\cdot) \in U_{2}^{1}$. Then it follows from (2.9) that

$$
\begin{gathered}
x_{k}(t)=\int_{0}^{t}-y_{k}^{2}(\tau) d \tau+\int_{0}^{t} u_{k}^{2}(\tau) d \tau, \\
y_{k}(t)=\int_{0}^{t} u_{k}(\tau) d \tau
\end{gathered}
$$

for every $t \in[0,1]$.

We get from (2.22) and (2.24) that

$$
y_{k}(t)= \begin{cases}t-\frac{2 i}{2 k}, & t \in\left[\frac{2 i}{2 k}, \frac{2 i+1}{2 k}\right) \\ -t+\frac{2 i+2}{2 k}, & t \in\left[\frac{2 i+1}{2 k}, \frac{2 i+2}{2 k}\right)\end{cases}
$$

for every $t \in[0,1]$ where $i=0,1, \ldots, k-1$.

Then, from (2.25) we have

$$
0 \leq y_{k}(t) \leq \frac{1}{2 k}
$$

for every $t \in[0,1]$, and consequently

$$
0 \leq y_{k}^{2}(t) \leq \frac{1}{4 k^{2}}
$$

for every $t \in[0,1]$.

According to (2.22), $u_{k}^{2}(t)=1$ for all $t \in[0,1]$. Then, from (2.23) and (2.27) we obtain that

$$
1-\frac{1}{4 k^{2}} \leq \dot{x}_{k}(t) \leq 1
$$

for almost all $t \in[0,1]$, and consequently

$$
\left(1-\frac{1}{4 k^{2}}\right) t \leq x_{k}(t) \leq t
$$

for every $t \in[0,1]$, where $k=1,2, \ldots$

We conclude from the last inequality that

$$
\left(1-\frac{1}{4 k^{2}}\right) \leq x_{k}(1) \leq 1
$$

for every $k=1,2, \ldots$

It follows from (2.26) and (2.30) that

$$
\left(x_{k}(1), y_{k}(1)\right) \longrightarrow(1,0) \text { as } k \longrightarrow \infty .
$$

Since $\left(x_{k}(1), y_{k}(1)\right) \in X_{2}(1 ; 0,(0,0), 1)$ for every $k=1,2, \ldots$, from (2.31) we obtain that

$$
(1,0) \in \operatorname{cl}\left(X_{2}(1 ; 0,(0,0), 1)\right) .
$$

However, (2.21) and (2.32) imply that $X_{2}(1 ; 0,(0,0), 1)$ is not a closed set. 


\section{Diameter of the attainable set and continuity with respect to $t$}

In this section we will give an upper estimation for the diameter of the attainable set $X_{p}\left(t ; t_{0}, X_{0}, \mu_{0}\right)$ and will show that the set-valued map $t \rightarrow X_{p}\left(t ; t_{0}, X_{0}, \mu_{0}\right)$ is Hölder continuous with respect to $t$.

We denote the diameter of a set $A \subset \mathbb{R}^{n}$ by $\operatorname{diam}(A)$ and define it as

$$
\operatorname{diam} A=\sup _{x, y \in A}\|x-y\|
$$

The following proposition characterizes the diameter of the attainable set $X_{p}\left(t ; t_{0}, X_{0}\right.$, $\left.\mu_{0}\right)$.

Proposition 3.1. For every $p \in(1,+\infty)$, the inequality

$$
\operatorname{diam} X_{p}\left(t ; t_{0}, X_{0}, \mu_{0}\right) \leq\left[d_{0}+r_{1}(t, p)\right] \exp \left(r_{0}(t, p)\right)
$$

holds for any $t \in\left[t_{0}, \theta\right]$, where

$$
\begin{gathered}
d_{0}=\operatorname{diam} X_{0}, \\
r_{0}(t, p)=L_{1}\left(t-t_{0}\right)+L_{2}\left(t-t_{0}\right)^{(p-1) / p}, \\
r_{1}(t, p)=2 L_{3} \mu_{0}\left(t-t_{0}\right)^{(p-1) / p} .
\end{gathered}
$$

Proof. Let $t \in\left[t_{0}, \theta\right]$ and $x_{1}(t) \in X_{p}\left(t ; t_{0}, X_{0}, \mu_{0}\right), x_{2}(t) \in X_{p}\left(t ; t_{0}, X_{0}, \mu_{0}\right)$ be arbitrarily chosen. Then there exist $x_{1} \in X_{0}, x_{1}(\cdot) \in X_{p}\left(t_{0}, x_{0}, \mu_{0}\right), u_{1}(\cdot) \in U_{p}^{\mu_{0}}, x_{2} \in X_{0}, x_{2}(\cdot) \in$ $X_{p}\left(t_{0}, x_{0}, \mu_{0}\right), u_{2}(\cdot) \in U_{p}^{\mu_{0}}$ such that

$$
\begin{aligned}
& x_{1}(t)=x_{1}+\int_{t_{0}}^{t} f\left(\tau, x_{1}(\tau), u_{1}(\tau)\right) d \tau, \\
& x_{2}(t)=x_{2}+\int_{t_{0}}^{t} f\left(\tau, x_{2}(\tau), u_{2}(\tau)\right) d \tau .
\end{aligned}
$$

Since $\left\|x_{1}-x_{2}\right\| \leq d_{0}$, then from (3.6), and the condition (b), we get

$$
\begin{aligned}
\left\|x_{1}(t)-x_{2}(t)\right\| \leq & d_{0}+\int_{t_{0}}^{t}\left(L_{1}+L_{2}\left\|u_{2}(\tau)\right\|\right)\left\|x_{1}(\tau)-x_{2}(\tau)\right\| d \tau \\
& +\int_{t_{0}}^{t} L_{3}\left\|u_{1}(\tau)-u_{2}(\tau)\right\| d \tau
\end{aligned}
$$

Since $u_{1}(\cdot), u_{2}(\cdot) \in U_{p}^{\mu_{0}}$, then the Hölder and Minkowski inequalities imply that

$$
L_{3} \int_{t_{0}}^{t}\left\|u_{1}(\tau)-u_{2}(\tau)\right\| d \tau \leq 2 L_{3} \mu_{0}\left(t-t_{0}\right)^{(p-1) / p}=r_{1}(t, p),
$$


where $r_{1}(t, p)$ is defined by (3.5). Since $t \in\left[t_{0}, \theta\right]$ is arbitrarily chosen, we obtain from (3.7), (3.8), and Gronwall's inequality that

$$
\begin{aligned}
\left\|x_{1}(t)-x_{2}(t)\right\| & \leq\left[d_{0}+r_{1}(t, p)\right] \exp \left(\int_{t_{0}}^{t}\left(L_{1}+L_{2}\left\|u_{2}(\tau)\right\|\right) d \tau\right) \\
& \leq\left[d_{0}+r_{1}(t, p)\right] \exp \left(L_{1}\left(t-t_{0}\right)+L_{2}\left(t-t_{0}\right)^{(p-1) / p} \mu_{0}\right) \\
& =\left[d_{0}+r_{1}(t, p)\right] \exp \left(r_{0}(t, p)\right),
\end{aligned}
$$

where $r_{0}(t, p)$ is defined by (3.4).

Note that an estimation for diameter of the attainable set can be obtained from Proposition 2.1; but the estimation given by Proposition 3.1 is more precise.

Corollary 3.2. $\operatorname{diam} X_{p}\left(t ; t_{0}, X_{0}, \mu_{0}\right) \rightarrow \operatorname{diam} X_{0}$ as $t \rightarrow t_{0}$.

The following proposition asserts that the attainable set $X_{p}\left(t ; t_{0}, X_{0}, \mu_{0}\right)$ is Hölder continuous with respect to $t$.

Proposition 3.3. Let $t_{1} \in\left[t_{0}, \theta\right], t_{2} \in\left[t_{0}, \theta\right]$. Then

$$
h\left(X_{p}\left(t_{1} ; t_{0}, X_{0}, \mu_{0}\right), X_{p}\left(t_{2} ; t_{0}, X_{0}, \mu_{0}\right)\right) \leq k_{*}\left|t_{1}-t_{2}\right|^{(p-1) / p},
$$

where $k_{*}>0$ is defined by (2.6).

Proof. Without loss of generality, let us assume that $t_{1}<t_{2}$. Let $y_{1} \in X_{p}\left(t_{1} ; t_{0}, X_{0}, \mu_{0}\right)$ be arbitrarily chosen. Then there exist $x_{0} \in X_{0}, x_{0}(\cdot) \in X_{p}\left(t_{0}, X_{0}, \mu_{0}\right)$ and $u_{0}(\cdot) \in U_{p}^{\mu_{0}}$ such that

$$
y_{1}=x_{0}\left(t_{1}\right)=x_{0}+\int_{t_{0}}^{t_{1}} f\left(\tau, x_{0}(\tau), u_{0}(\tau)\right) d \tau \text {. }
$$

Let

$$
y_{2}=x_{0}\left(t_{2}\right)=x_{0}+\int_{t_{0}}^{t_{2}} f\left(\tau, x_{0}(\tau), u_{0}(\tau)\right) d \tau \text {. }
$$

It is obvious that $y_{2} \in X_{p}\left(t_{2} ; t_{0}, X_{0}, \mu_{0}\right)$. From Proposition 2.1, relations (2.4), (2.6), (3.11), (3.12), and the condition (c), we have

$$
\begin{aligned}
\left\|y_{1}-y_{2}\right\| & \leq \int_{t_{1}}^{t_{2}} c\left(1+\left\|x_{0}(\tau)\right\|\right)\left(1+\left\|u_{0}(\tau)\right\|\right) d \tau \\
& \leq c\left(1+r_{*}\right) \int_{t_{1}}^{t_{2}}\left(1+\left\|u_{0}(\tau)\right\|\right) d \tau \\
& \leq c\left(1+r_{*}\right)\left(\left|t_{2}-t_{1}\right|+\mu_{0}\left|t_{2}-t_{1}\right|^{(p-1) / p}\right) \\
& \left.\leq c\left(1+r_{*}\right)\left(l_{*}+\mu_{0}\right)\left|t_{2}-t_{1}\right|^{(p-1) / p}\right)=k_{*}\left|t_{2}-t_{1}\right|^{(p-1) / p},
\end{aligned}
$$

where $c>0$ is the constant given in condition (c). 
Since $y_{1} \in X_{p}\left(t_{1}, t_{0}, X_{0}, \mu_{0}\right)$ is arbitrarily chosen, then (3.13) implies that

$$
X_{p}\left(t_{1} ; t_{0}, X_{0}, \mu_{0}\right) \subset X_{p}\left(t_{2} ; t_{0}, X_{0}, \mu_{0}\right)+k_{*}\left|t_{2}-t_{1}\right|^{(p-1) / p} B_{n}(1) .
$$

Analogously, it is possible to show that

$$
X_{p}\left(t_{2} ; t_{0}, X_{0}, \mu_{0}\right) \subset X_{p}\left(t_{1} ; t_{0}, X_{0}, \mu_{0}\right)+k_{*}\left|t_{2}-t_{1}\right|^{(p-1) / p} B_{n}(1) .
$$

In fact, (3.14) and (3.15) yield the proof.

From Proposition 3.3, we obtain the following corollary.

Corollary 3.4. The set-valued map $t \rightarrow X_{p}\left(t ; t_{0}, X_{0}, \mu_{0}\right), t \in\left[t_{0}, \theta\right]$, is $(p-1) / p$-Hölder continuous.

\section{Dependence of the attainable sets on parameters $t_{0}$ and $X_{0}$}

The following proposition characterizes the continuity of the set-valued map $\left(t_{0}, X_{0}\right) \rightarrow$ $X_{p}\left(t ; t_{0}, X_{0}, \mu_{0}\right)$ in the Hausdorff metric.

Let us denote

$$
\omega_{*}=\left(L_{1}+L_{2} \mu_{0}\right) l_{*}
$$

where $l_{*}$ is defined by (2.4), $L_{1}$ and $L_{2}$ are the constants given in condition (b).

Proposition 4.1. Let $t_{1} \geq t_{0}$ and $X_{0}, X_{1} \subset \mathbb{R}^{n}$ be compact sets. Then the inequality

$$
h\left(X_{p}\left(t ; t_{0}, X_{0}, \mu_{0}\right), X_{p}\left(t ; t_{1}, X_{1}, \mu_{0}\right)\right) \leq\left[h\left(X_{0}, X_{1}\right)+\left(t_{1}-t_{0}\right)^{(p-1) / p} k_{*}\right] \exp \left(\omega_{*}\right)
$$

holds for all $t \in\left[t_{1}, \theta\right]$, where $k_{*}$ is defined by (2.6), $\omega_{*}$ is defined by (4.1).

Proof. Let us choose arbitrary $t \in\left[t_{1}, \theta\right]$ and $x_{0}(t) \in X_{p}\left(t ; t_{0}, X_{0}, \mu_{0}\right)$, where $x_{0}(\cdot) \in$ $X_{p}\left(t_{0}, X_{0}, \mu_{0}\right)$. Then there exist $x_{0} \in X_{0}$ and $u_{0}(\cdot) \in U_{p}^{\mu_{0}}$ such that

$$
x_{0}(t)=x_{0}+\int_{t_{0}}^{t} f\left(\tau, x_{0}(\tau), u_{0}(\tau)\right) d \tau
$$

holds. According to the definition of Hausdorff distance, there exists $x_{1} \in X_{1}$ such that $\| x_{1}-$ $x_{0} \| \leq h\left(X_{0}, X_{1}\right)$. Let $x_{1}(\cdot)$ be a solution of the control system (1.1), generated by the admissible control function $u_{0}(\cdot)$ with initial condition $x_{1}\left(t_{1}\right)=x_{1} \in X_{1}$. Then

$$
x_{1}(t)=x_{1}+\int_{t_{1}}^{t} f\left(\tau, x_{1}(\tau), u_{0}(\tau)\right) d \tau
$$

and $x_{1}(t) \in X_{p}\left(t ; t_{1}, X_{1}, \mu_{0}\right)$.

From (4.3), (4.4), and conditions (b) and (c), we have

$$
\begin{aligned}
\left\|x_{0}(t)-x_{1}(t)\right\| \leq & h\left(X_{0}, X_{1}\right)+c \int_{t_{0}}^{t_{1}}\left(1+\left\|u_{0}(\tau)\right\|\right)\left(1+\left\|x_{0}(\tau)\right\|\right) d \tau \\
& +\int_{t_{1}}^{t}\left(L_{1}+L_{2}\left\|u_{0}(\tau)\right\|\right)\left(\left\|x_{0}(\tau)-x_{1}(\tau)\right\|\right) d \tau .
\end{aligned}
$$


Proposition 2.1 implies that

$$
c \int_{t_{0}}^{t_{1}}\left(1+\left\|u_{0}(\tau)\right\|\right)\left(1+\left\|x_{0}(\tau)\right\|\right) d \tau \leq\left(t_{1}-t_{0}\right)^{(p-1) / p} k_{* \prime}
$$

where $k_{*}$ is defined by (2.6). Since $t \in\left[t_{1}, \theta\right]$ is arbitrarily chosen, from (4.5), (4.6), and Gronwall's inequality, we get

$$
\begin{aligned}
\left\|x_{0}(t)-x_{1}(t)\right\| & \leq\left[h\left(X_{0}, X_{1}\right)+\left(t_{1}-t_{0}\right)^{(p-1) / p} k_{*}\right] \exp \left(\int_{t_{1}}^{t}\left(L_{1}+L_{2}\left\|u_{0}(\tau)\right\|\right) d \tau\right) \\
& \leq\left[h\left(X_{0}, X_{1}\right)+\left(t_{1}-t_{0}\right)^{(p-1) / p} k_{*}\right] \exp \left(\omega_{*}\right) .
\end{aligned}
$$

Hence, we obtain from (4.7) that

$$
X_{p}\left(t ; t_{0}, X_{0}, \mu_{0}\right) \subset X_{p}\left(t ; t_{1}, X_{1}, \mu_{0}\right)+\left[h\left(X_{0}, X_{1}\right)+\left(t_{1}-t_{0}\right)^{(p-1) / p} k_{*}\right] \exp \left(\omega_{*}\right) B_{n}(1) .
$$

Similarly, one can prove that

$$
X_{p}\left(t ; t_{1}, X_{1}, \mu_{0}\right) \subset X_{p}\left(t ; t_{0}, X_{0}, \mu_{0}\right)+\left[h\left(X_{0}, X_{1}\right)+\left(t_{1}-t_{0}\right)^{(p-1) / p} k_{*}\right] \exp \left(\omega_{*}\right) B_{n}(1) .
$$

Finally, (4.8) and (4.9) complete the proof.

From Proposition 4.1, the validity of the following corollaries follow.

Corollary 4.2. The inequality

$$
h\left(X_{p}\left(t ; t_{0}, X_{0}, \mu_{0}\right), X_{p}\left(t ; t_{0}, X_{1}, \mu_{0}\right)\right) \leq h\left(X_{0}, X_{1}\right) \exp \left(\omega_{*}\right)
$$

holds for all $t \in\left[t_{0}, \theta\right]$, where $\omega_{*}>0$ is defined by (4.1).

Corollary 4.3. The inequality

$$
h_{C}\left(X_{p}\left(t_{0}, X_{0}, \mu_{0}\right), X_{p}\left(t_{0}, X_{1}, \mu_{0}\right)\right) \leq h\left(X_{0}, X_{1}\right) \exp \left(\omega_{*}\right)
$$

holds.

Corollary 4.4. Let $X_{0} \subset \mathbb{R}^{n}$ and $X_{n} \subset \mathbb{R}^{n}$ be compact sets for all $n=1,2, \ldots$. Assume that $h\left(X_{n}, X_{0}\right) \rightarrow 0$ and $t_{n} \rightarrow t_{0}+0$ as $n \rightarrow \infty$. Then for all $t \in\left(t_{0}, \theta\right]$,

$$
h\left(X_{p}\left(t ; t_{n}, X_{n}, \mu_{0}\right), X_{p}\left(t ; t_{0}, X_{0}, \mu_{0}\right)\right) \longrightarrow 0 \text { as } n \longrightarrow \infty \text {. }
$$

\section{Dependence of the attainable sets on $\mu_{0}$}

In this section we specify dependence of the set $X_{p}\left(t_{0}, X_{0}, \mu_{0}\right)$ on the constraint parameter $\mu_{0}$. Let

$$
r_{1}=L_{3} l_{*} \exp \left(\omega_{*}\right),
$$

where $\omega_{*}$ is defined by (4.1).

The following proposition characterizes the relation between the solutions sets $X_{p}\left(t_{0}\right.$, $\left.X_{0}, \mu_{*}\right)$ and $X_{p}\left(t_{0}, X_{0}, \mu_{0}\right)$. 
Proposition 5.1. The inequality

$$
h_{C}\left(X_{p}\left(t_{0}, X_{0}, \mu_{*}\right), X_{p}\left(t_{0}, X_{0}, \mu_{0}\right)\right) \leq r_{1}\left|\mu_{*}-\mu_{0}\right|
$$

is satisfied, where $r_{1}$ is defined by (5.1).

Proof. Let $x_{0}(\cdot) \in X_{p}\left(t_{0}, X_{0}, \mu_{0}\right)$ be an arbitrarily chosen solution. Then there exist $x_{0} \in X_{0}$ and $u_{0}(\cdot) \in U_{p}^{\mu_{0}}$ such that

$$
x_{0}(t)=x_{0}+\int_{t_{0}}^{t} f\left(\tau, x_{0}(\tau), u_{0}(\tau)\right) d \tau
$$

for every $t \in\left[t_{0}, \theta\right]$.

We define a new control function $u_{*}(\cdot):\left[t_{0}, \theta\right] \rightarrow \mathbb{R}^{m}$, setting

$$
u_{*}(t)=\frac{\mu_{*}}{\mu_{0}} u_{0}(t), \quad t \in\left[t_{0}, \theta\right]
$$

It is not difficult to verify that $u_{*}(\cdot) \in U_{p}^{\mu_{*}}$. Let $x_{*}(\cdot)$ be a solution of the control system (1.1), generated by $u_{*}(\cdot) \in U_{p}^{\mu_{*}}$ from the initial point $\left(t_{0}, x_{0}\right)$. Then $x_{*}(\cdot) \in X_{p}\left(t_{0}, X_{0}\right.$, $\left.\mu_{*}\right)$ and

$$
x_{*}(t)=x_{0}+\int_{t_{0}}^{t} f\left(\tau, x_{*}(\tau), u_{*}(\tau)\right) d \tau
$$

for every $t \in\left[t_{0}, \theta\right]$. From (5.3), (5.4), (5.5), and condition (b), we get

$$
\begin{aligned}
\left\|x_{*}(t)-x_{0}(t)\right\| & \leq L_{3}\left|\frac{\mu_{*}}{\mu_{0}}-1\right| \int_{t_{0}}^{t}\left\|u_{0}(\tau)\right\| d \tau+\int_{t_{0}}^{t}\left(L_{1}+L_{2}\left\|u_{0}(\tau)\right\|\right)\left\|x_{*}(\tau)-x_{0}(\tau)\right\| d \tau \\
& \leq L_{3} l_{*}\left|\mu_{*}-\mu_{0}\right|+\int_{t_{0}}^{t}\left(L_{1}+L_{2}\left\|u_{0}(\tau)\right\|\right)\left\|x_{*}(\tau)-x_{0}(\tau)\right\| d \tau
\end{aligned}
$$

for every $t \in\left[t_{0}, \theta\right]$, where $l_{*}$ is defined by (2.4).

The Gronwall inequality, (5.1), and (5.6) yield that

$$
\begin{aligned}
\left\|x_{*}(t)-x_{0}(t)\right\| & \leq L_{3} l_{*}\left|\mu_{*}-\mu_{0}\right| \exp \left(L_{1}\left(\theta-t_{0}\right)+L_{2} \mu_{0}\left(\theta-t_{0}\right)^{(p-1) / p}\right) \\
& \leq L_{3} l_{*} \exp \left(\omega_{*}\right)\left|\mu_{*}-\mu_{0}\right|=r_{1}\left|\mu_{*}-\mu_{0}\right|
\end{aligned}
$$

for all $t \in\left[t_{0}, \theta\right]$.

Thus from (5.7) we get that for any fixed $x_{0}(\cdot) \in X_{p}\left(t_{0}, X_{0}, \mu_{0}\right)$ there exists $x_{1}(\cdot) \in$ $X_{p}\left(t_{0}, X_{0}, \mu_{*}\right)$ such that

$$
\left\|x_{0}(\cdot)-x_{*}(\cdot)\right\|_{C} \leq r_{1}\left|\mu_{*}-\mu_{0}\right|
$$

and consequently

$$
X_{p}\left(t_{0}, X_{0}, \mu_{0}\right) \subset X_{p}\left(t_{0}, X_{0}, \mu_{*}\right)+r_{1}\left|\mu_{*}-\mu_{0}\right| B_{C},
$$

where $B_{C}$ is the closed unit ball centered at the origin in the space $C\left(\left[t_{0}, \theta\right], \mathbb{R}^{n}\right)$.

Analogously, it is possible to prove that

$$
X_{p}\left(t_{0}, X_{0}, \mu_{*}\right) \subset X_{p}\left(t_{0}, X_{0}, \mu_{0}\right)+r_{1}\left|\mu_{*}-\mu_{0}\right| B_{C} .
$$

Hence, from (5.9) and (5.10), we obtain the proof of the proposition. 
From Proposition 5.1, it follows that the following corollaries are satisfied.

Corollary 5.2. The inequality

$$
h\left(X_{p}\left(t ; t_{0}, X_{0}, \mu_{*}\right), X_{p}\left(t ; t_{0}, X_{0}, \mu_{0}\right)\right) \leq r_{1}\left|\mu_{*}-\mu_{0}\right|
$$

is satisfied for any $t \in\left[t_{0}, \theta\right]$, where $r_{1}>0$ is defined by (5.1).

Corollary 5.3. Let $\mu_{n} \rightarrow \mu_{0}$ as $n \rightarrow \infty$. Then

$$
\begin{gathered}
h_{C}\left(X_{p}\left(t_{0}, X_{0}, \mu_{n}\right), X_{p}\left(t_{0}, X_{0}, \mu_{0}\right)\right) \longrightarrow 0 \text { as } n \longrightarrow \infty, \\
h\left(X_{p}\left(t ; t_{0}, X_{0}, \mu_{n}\right), X_{p}\left(t ; t_{0}, X_{0}, \mu_{0}\right)\right) \longrightarrow 0 \text { as } n \longrightarrow \infty
\end{gathered}
$$

for every $t \in\left[t_{0}, \theta\right]$.

\section{Acknowledgments}

This research was supported by the Scientific and Technological Research Council of Turkey (TUBITAK) Project no. 106T012. The authors thank the referees for the careful reading of the manuscript and the helpful suggestions.

\section{References}

[1] V. V. Beletskii, Studies of Motions of Celestial Bodies, Nauka, Moscow, Russia, 1972.

[2] N. N. Krasovskii, Theory of Control of Motion: Linear Systems, Nauka, Moscow, Russia, 1968.

[3] V. I. Ukhobotov, One Dimensional Projection Method in Linear Differential Games with Integral Constraints, Chelyabinsk State University Press, Chelyabinsk, Russia, 2005.

[4] A. G. Chentsov, "Asymptotic attainability with perturbation of integral constraints in an abstract control problem-I," Russian Mathematics, vol. 39, no. 2, pp. 57-68, 1995.

[5] A. G. Chentsov, Asymptotic Attainability, Kluwer Academic Publishers, Dordrecht, The Netherlands, 1997.

[6] R. Conti, Problemi di Controllo e di Controllo Ottimale, UTET, Torino, Italy, 1974.

[7] F. Gozzi and P. Loreti, "Regularity of the minimum time function and minimum energy problems: the linear case," SIAM Journal on Control and Optimization, vol. 37, no. 4, pp. 1195-1221, 1999.

[8] H. W. Lou, "On the attainable sets of control systems with p-integrable controls," Journal of Optimization Theory and Applications, vol. 123, no. 1, pp. 123-147, 2004.

[9] M. Motta and C. Sartori, "Minimum time and minimum energy function for linear systems with controls in $L^{p}$ for $p \geq 1$," preprint, 2000.

[10] A. N. Sirotin and A. M. Formalskii, "Reachability and controllability of discrete-time systems under control actions bounded in magnitude and norm," Automation and Remote Control, vol. 64, no. 12, pp. 1844-1857, 2003.

[11] Kh. G. Guseľnov, A. A. Neznakhin, and V. N. Ushakov, “Approximate construction of reachable sets of control systems with integral constraints on the controls," Journal of Applied Mathematics and Mechanics, vol. 63, no. 4, pp. 557-567, 1999.

[12] Kh. G. Guseinov, O. Ozer, and E. Akyar, "On the continuity properties of the attainable sets of control systems with integral constraints on control," Nonlinear Analysis: Theory, Methods E Applications, vol. 56, no. 3, pp. 433-449, 2004.

[13] Kh. G. Guseinov, O. Ozer, E. Akyar, and V. N. Ushakov, “The approximation of reachable sets of control systems with integral constraint on controls," Nonlinear Differential Equations and Applications, vol. 14, no. 1-2, pp. 57-73, 2007.

[14] M. Motta and C. Sartori, "Minimum time with bounded energy, minimum energy with bounded time," SIAM Journal on Control and Optimization, vol. 42, no. 3, pp. 789-809, 2003. 
[15] Kh. G. Guseinov and A. S. Nazlipinar, "On the continuity property of $L_{p}$ balls and an application," Journal of Mathematical Analysis and Applications, vol. 335, no. 2, pp. 1347-1359, 2007.

[16] M. Motta and F. Rampazzo, "Multivalued dynamics on a closed domain with absorbing boundary. Applications to optimal control problems with integral constraints," Nonlinear Analysis: Theory, Methods E Applications, vol. 41, no. 5-6, pp. 631-647, 2000.

[17] B. T. Polyak, "Convexity of the reachable set of nonlinear systems under $L_{2}$ bounded controls," Tech. Rep. 02.2002/2003, Institut Mittag-Leffler, Djursholm, Sweden, 2003.

[18] P. Soravia, "Viscosity solutions and optimal control problems with integral constraints," Systems $\mathcal{E}$ Control Letters, vol. 40, no. 5, pp. 325-335, 2000.

[19] A. F. Filippov, "On some questions in the theory of optimal regulation: existence of a solution of the problem of optimal regulation in the class of bounded measurable functions," Vestnik Moskovskogo Universiteta, Seriya Matematika, Mekhanika, vol. 1959, no. 2, pp. 25-32, 1959.

[20] A. F. Filippov, Differential Equations with Discontinuous Righthand Sides, vol. 18 of Mathematics and Its Applications, Kluwer Academic Publishers, Dordrecht, The Netherlands, 1988. 


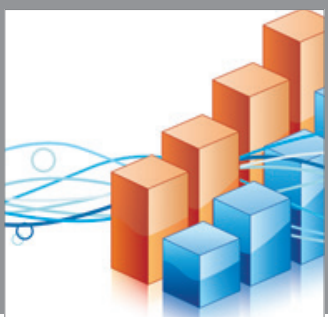

Advances in

Operations Research

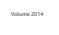

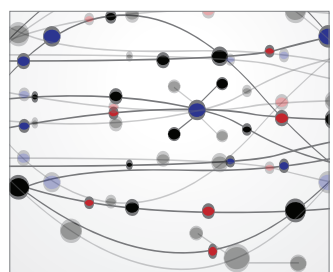

\section{The Scientific} World Journal
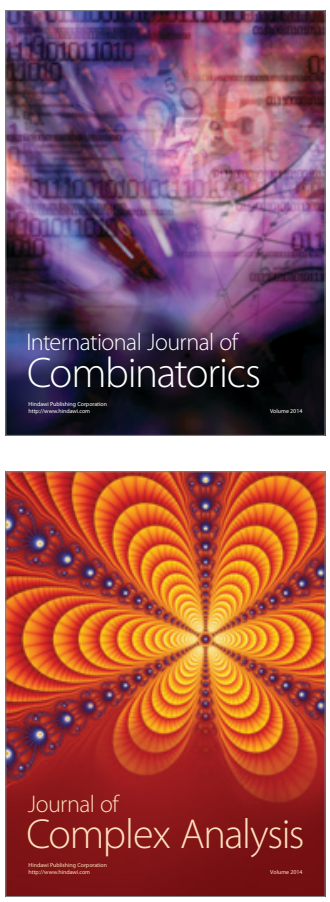

International Journal of

Mathematics and

Mathematical

Sciences
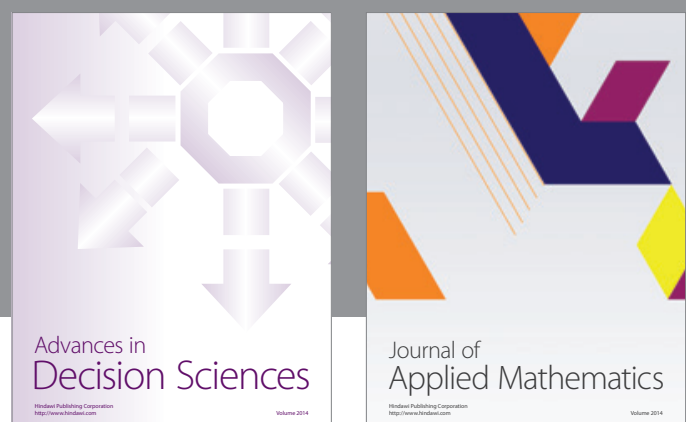

Journal of

Applied Mathematics
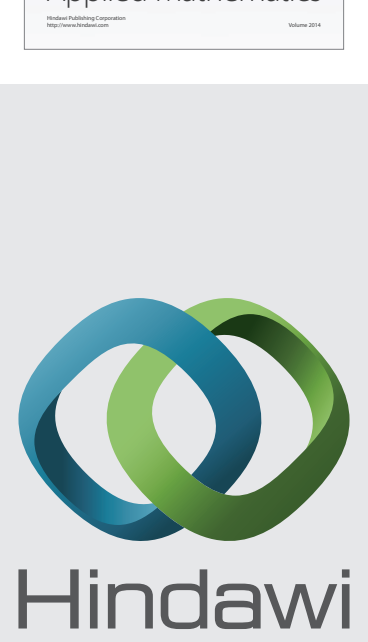

Submit your manuscripts at http://www.hindawi.com
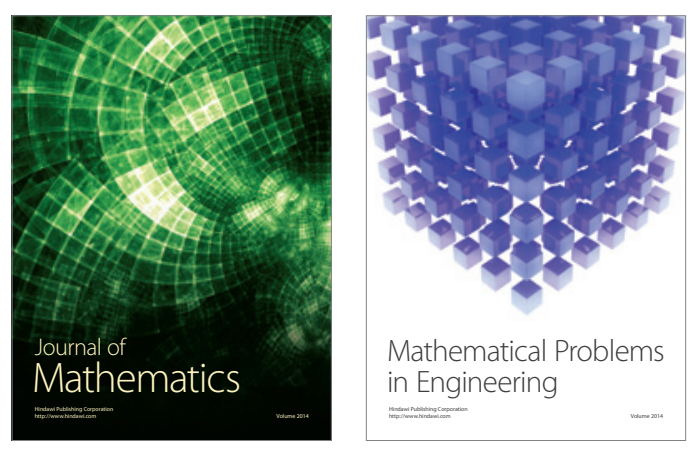

Mathematical Problems in Engineering
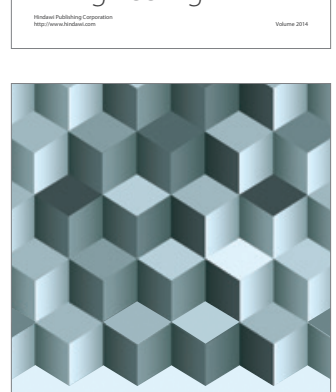

Journal of

Function Spaces
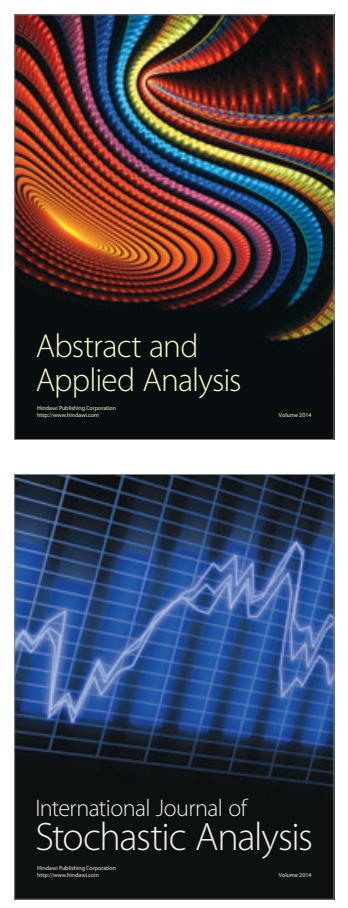

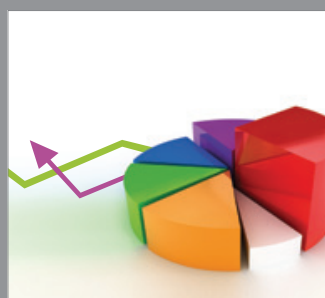

ournal of

Probability and Statistics

Promensencen
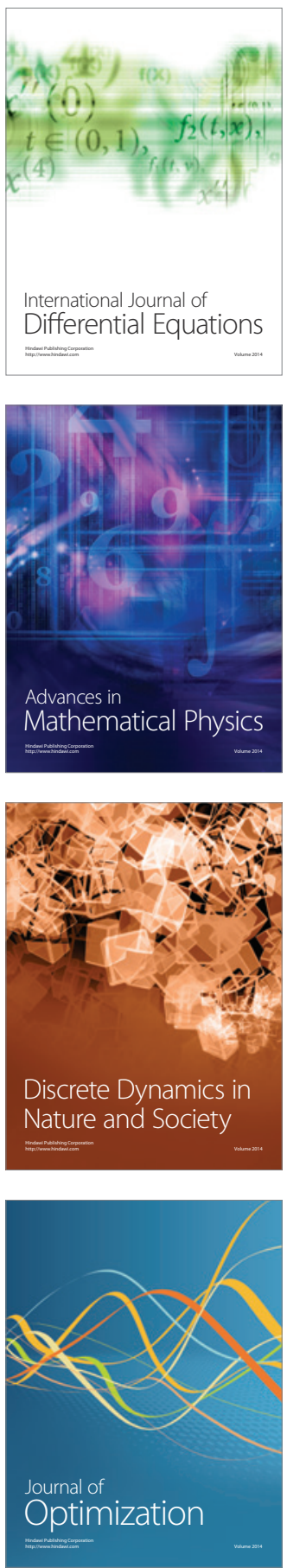